\title{
Rol del fisioterapeuta y la importancia de la educación sexual integral en la mujer
}

Physiotherapist role and the importance of comprehensive sex education in women

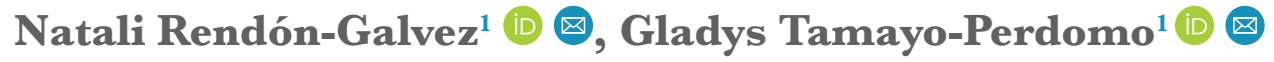 \\ ${ }^{1}$ Programa de Fisioterapia; Facultad de Ciencias de la Salud; Fundación Universitaria María Cano; Neiva; Colombia.
}

\section{D) Check for updates}

\section{Correspondencia}

Natali Rendón-Galvez.

Email: natalirendongalvez@fumc.edu.co

\section{Gitar así \\ Rendón-Galvez, Natali; Tamayo-Perdomo, Gladys. (2022). Rol del fisioterapeuta y la importancia de la educación sexual integral en la mujer. Revista de Investigación e Innovación en Ciencias de la Salud. 4(1), 125-136. https://doi. org/ 10.46634/riics. 82}

Recibido: $14 / 09 / 2021$

Revisado: $27 / 10 / 202$

Aceptado: 09/11/2021

\section{Editor}

Jorge Mauricio Cuartas Arias, Ph.D.

\section{Coeditor}

Fraidy-Alonso Alzate-Pamplona, MSc.

Gopyright $($ ) 2022. Fundación Universitaria María Cano. La Revista de Investigación e

Innovación en Ciencias de la Salud proporciona acceso abierto a todo su contenido bajo los términos de la licencia Creative Commons Attribution-NonCommercial-NoDerivatives 4.0 International (CG BY-NG-ND 4.0).

\section{Declaración de intereses \\ Las autoras han declarado que no hay conflicto de intereses.}

\section{Disponibilidad de datos}

Todos los datos relevantes se encuentran en el artículo. Para mayor información, comunicarse con el autor de correspondencia.

\section{Resumen}

Introducción. Colombia es un país que enfrenta diversas situaciones provenientes de la falta de educación sexual y educación en salud adecuada: casos de abuso sexual, embarazos en niñas menores de catorce años y la incidencia y prevalencia de la incontinencia urinaria por afectación del piso pélvico.

Objetivo. El presente artículo busca dar cuenta del desconocimiento en temas de salud sexual y sus consecuencias, evidenciar los beneficios que podrían generar las intervenciones de educación sexual integral en el país y reflexionar sobre el rol del fisioterapeuta en la rehabilitación y prevención de disfunciones del piso pélvico en las mujeres.

Reflexión. Si se incorporaran procesos de fisioterapia pélvica como componente preventivo, que incluya educación, se podría reducir el desconocimiento, el riesgo de vulneración de los derechos sexuales y reproductivos y futuras disfunciones del piso pélvico. La importancia de la educación desde la fisioterapia radica en que las mujeres reconozcan que, al aumentar la fuerza de su musculatura, están cuidando de su piso pélvico hasta edades avanzadas, en las que se estima que la prevalencia de incontinencia urinaria es del $34 \%$.

Conclusiones: Un programa de educación sexual impartido desde el currículo podría disminuir las condiciones de salud del piso pélvico, así como los factores de riesgo de abuso sexual. Además, permitiría el acompañamiento médico adecuado para orientar el inicio de la vida sexual en un proceso seguro y consciente, así como la expresión de la sexualidad con libertad, al ser concebida como un derecho.

\section{Palabras clave}

Sexualidad; educación sexual; derechos sexuales y reproductivos; salud sexual y reproductiva; educación en salud; promoción de la salud; prevención de enfermedades; piso pélvico; fisioterapia; síntomas del sistema urinario inferior; mujeres. 


\section{Financiamiento}

Departamento Administrativo de Ciencia, Tecnología E Innovación - Colciencias. Convocatoria $\mathrm{N}^{\circ}$ 856 Jóvenes Investigadores e Innovadores para el Departamento del Huila - 2019.

\section{Descargo de responsabilidad El contenido de este artículo es responsabilidad exclusiva de los autores y no representa una opinión oficial de sus instituciones ni de la Revista de Investigación e Innovación en Ciencias de la Salud.}

Contribución de los autores

Natali Rendón-Galvez: conceptualización, curación de datos, análisis formal, adquisición de fondos, investigación, metodología, administración de proyecto, recursos, software, supervisión, validación, visualización, escritura: borrador original, escritura: revisión y edición. Gladys Tamayo-Perdomo: conceptualización, curación de datos, análisis formal, investigación, metodología, supervisión, validación, visualización, escritura: borrador original, escritura: revisión y edición.

\section{Abstract}

Introduction. Colombia is a country that faces various situations stemming from the lack of sexual education and adequate health education: cases of sexual abuse, pregnancies in girls under fourteen years old, and the incidence and prevalence of urinary incontinence due to pelvic floor involvement.

Aim. This article seeks to account for the lack of knowledge about sexual health issues and their consequences. Also, to show the benefits that comprehensive sexuality education interventions could generate in the country. Finally, to reflect on the physiotherapist's role in the rehabilitation and prevention of pelvic floor dysfunctions in women.

Reflection. If pelvic physiotherapy processes were incorporated as a preventive component, including sexual education, it could reduce ignorance, the risk of violation of sexual and reproductive rights, and future dysfunctions of the pelvic floor. The importance of physiotherapy's education is that women recognize that, by increasing the strength of their muscles, they are taking care of their pelvic floor until advanced ages, in which it is estimated that the prevalence of urinary incontinence is $34 \%$.

Conclusions. A sexual education program taught from the curriculum could reduce the health conditions of the pelvic floor, as well as the risk factors for sexual abuse. It would also allow adequate medical support to guide the beginning of sexual life in a safe and conscious process, as well as the expression of sexuality with freedom as it is conceived as a right.

\section{Keywords}

Sexuality; sex education; reproductive rights; sexual and reproductive health; health education; health promotion; disease prevention; pelvic floor; physical therapy, lower urinary tract symptoms; women.

\section{Introducción}

Colombia es un país donde se realizan en promedio 43 dictámenes diarios de abuso sexual a menores [1]; en el que casi el $40 \%$ de los menores de quince años ya ha iniciado su vida sexual [2]; hay además 2.348 casos de nacimientos en niñas menores de catorce años entre enero y julio de 2020, donde el padre era mayor de veinte años en el 16\% de estos, y que, hasta septiembre de 2021, reportó 2.573 casos de nacimientos de madres menores de catorce años [3]. En ese sentido, es en un país golpeado por el desconocimiento y la estigmatización, en el que se hace más que evidente la necesidad de acciones que ayuden a disminuir estas estadísticas.

La problemática no se queda ahí, ya que, debido a la falta de educación en salud, no solo en etapas tempranas, sino en las edades siguientes, como la adolescencia y la adultez, se evidencian trastornos y disfunciones uroginecológicas que en ocasiones son prevenibles.

Con el objetivo de preparar a los niños, niñas y adolescentes (NNA) con conocimientos, habilidades, actitudes y valores que los empoderen para realizar su salud, bienestar y dignidad, desarrollar relaciones sociales y sexuales respetuosas y entender cuáles son sus derechos y asegurarse de protegerlos durante todo su ciclo vital [4-6], 
se desarrolla la Educación Sexual Integral (ESI), la cual es definida por la UNESCO y la OMS como un proceso que cuenta con un currículo de enseñanza para dar a conocer la sexualidad desde aspectos cognitivos, emocionales, físicos y sociales.

Además, la ESI considera la sexualidad de manera integral, debe ser basada en evidencias, bajo bases científicas, impartida con contenidos adecuados para la etapa en la que se encuentre cada persona, e incluir el desarrollo de habilidades reforzadas con elementos teóricos [6].

De acuerdo a lo anterior, el presente artículo tiene como propósito dar cuenta de las situaciones ocasionadas por la ignorancia en temas de salud sexual, la estigmatización cultural de la sexualidad, acompañado de problemáticas sociales, y, por lo tanto, evidenciar los beneficios que podrían generar las intervenciones de educación sexual integral en el país.

Así mismo, busca reflexionar sobre el rol del fisioterapeuta y sus acciones de promoción y educación en salud, la prevención de disfunciones de piso pélvico en las mujeres y su rehabilitación, haciendo énfasis en la incontinencia urinaria, al ser una de las condiciones con mayor incidencia, que se convierte en un problema higiénico y social para las mujeres que la padecen, influyendo en la calidad de vida, además de afectar su relación de pareja y sexualidad [7].

Estas reflexiones se generan desde los resultados del proyecto de investigación "Efectos de la fisioterapia de piso pélvico en la calidad de vida y en los factores biomecánicos de la marcha en mujeres con incontinencia urinaria", y que fue avalado y financiado por el Ministerio de Ciencia, Tecnología e Innovación, en la Convocatoria 856, Jóvenes Investigadores e Innovadores para el departamento del Huila.

En el mencionado proyecto de investigación, aprobado por el Comité de ética de la Fundación Universitaria María Cano, se llevó a cabo un protocolo de educación, basado en el concepto de las 5F's, propuesta por Bary Berhgmans [8], y de acuerdo a las recomendaciones previas al inicio de un plan de entrenamiento de los músculos del piso pélvico, brindadas por Kari Bø y Siv Mørkved [9]. Este protocolo de educación incluyó temas sobre anatomía, fisiología, fisiopatología, tratamiento, beneficios de la fisioterapia y hábitos de vida saludable, además de un protocolo de entrenamiento de los músculos del piso pélvico.

La valoración de las participantes de la investigación incluyó, además de los datos clínicos, la evaluación de la calidad de vida a través del instrumento ICIQ-LUTSqol-Spanish, de manera previa y posterior a la intervención con el protocolo, el cual fue aplicado a seis mujeres con un promedio de edad de 44 años, diagnosticadas con incontinencia urinaria de esfuerzo.

Tras el análisis inicial de los datos, se encontró que las mujeres participantes refirieron presentar afectación a nivel de las actividades de la vida diaria, sus relaciones de pareja y vida sexual. Así mismo, se logró identificar el desconocimiento sobre su condición de salud, causas y tratamiento de la misma. De igual manera, se evidenciaron mitos sobre la salud de la mujer, infundados en su entorno sociofamiliar.

En la evaluación final, las usuarias coincidieron en que se sentían más tranquilas y confiadas en sí mismas después de recibir las sesiones de educación. Debido a lo anterior, y de acuerdo a los antecedentes que demuestran la implicación de la falta de conocimiento por parte de las mujeres [10-12], se desarrolla el presente artículo. 
A continuación, se despliegan una serie de tópicos, a través del ciclo vital de la mujer, para evidenciar cómo, por medio de la educación como acción preventiva, se puede generar un fortalecimiento del derecho al goce de una sexualidad saludable, evitando condiciones de salud del tracto urinario bajo, y cómo la fisioterapia participa activamente en este proceso.

\section{Reflexión}

\section{Educación sexual integral, ¿una necesidad en Colombia?}

Colombia cuenta con legislación en relación a la educación sexual [13-16], que tiene como objetivos garantizar el desarrollo de una sana sexualidad y respeto mutuo, impartir la educación sexual en las instituciones educativas de acuerdo a la edad de los educandos, prevenir la violencia sexual en el nivel territorial, la promoción y la garantía de los derechos sexuales y la disminución de embarazos en la adolescencia, así como las enfermedades de transmisión sexual. Pero, a pesar de la existencia de estas leyes y decretos, se evidencia una carencia de estrategias que permitan establecer una cátedra dedicada a la educación sexual, y en cambio es desarrollada de forma transversal con demás asignaturas del currículo o, a través de actividades extracurriculares.

Un ejemplo claro de la normatividad es la Ley 1146 de 2017, por medio de la cual se expiden normas para la prevención de la violencia sexual y atención integral de los niños, niñas y adolescentes abusados sexualmente [14]. Al respecto, algunos magistrados han fallado en contra, debido principalmente a que en el artículo 14 se realiza una exclusión de la cátedra de educación para la sexualidad en la educación básica primaria [17].

La falencia de una estructuración de esta educación sexual en las instituciones educativas, y en especial la excepción de ciertas edades, puede fortalecer acciones en las que los NNA sean vulnerados en sus derechos sexuales y reproductivos. Según los boletines estadísticos mensuales emitidos por el Instituto Nacional de Medicina Legal y Ciencias Forenses, el grupo de edad en el que más se realizan exámenes médicolegales por presunto delito sexual, y por lo tanto presentan mayor riesgo de ser víctimas de algún delito sexual, es entre los diez y catorce años. Para el 2015, 8.602 niños y niñas en este rango de edad pasaron por dicho examen, y la cifra fue de 7.257 exámenes en el 2020, [1].

De acuerdo a la UNESCO, la primera infancia abarca la edad de los cero a los ocho años, edades en las que los niños y niñas colombianos se encuentran cursando los niveles escolares de básica primaria, además de tratarse del grupo que se encuentra en el segundo puesto de vulnerabilidad de acuerdo a la misma fuente [1], presentando para el 2015 un total de 5.431 exámenes médico-legales realizados en niños y niñas entre los cinco a nueve años por presunto abuso sexual, y 4.063 exámenes realizados en 2020 (ver Figura 1).

Adicional a lo anterior, se evidencia que los niños, niñas y adolescentes no están recibiendo un correcto seguimiento y se encuentran expuestos a prácticas no seguras. De acuerdo a un estudio realizado por el DANE en el 2014, con 99.910 NNA de educación secundaria, el $39,4 \%$ de los menores de quince años habían iniciado su vida sexual, y del total de mujeres que refirieron haberla iniciado, un $26,1 \%$ respondió que su primera experiencia sexual fue con alguien mayor a ellas (cinco años o más) [2]. 


\section{Exámenes médicolegales por presunto delito sexual por año y rango de edad}

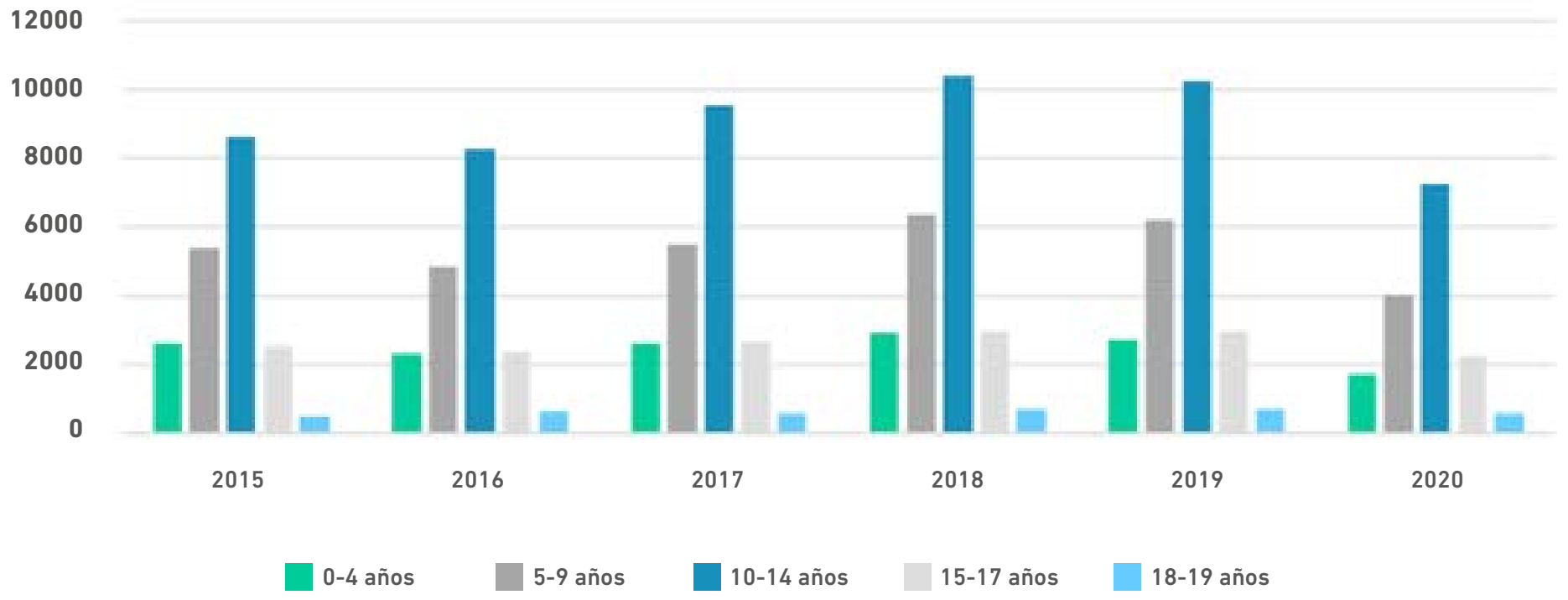

Figura 1. Exámenes medicolegales por presunto delito sexual por año y rango de edad

Nota. Gráfico desarrollado a partir de los datos obtenidos de los Boletines Estadísticos Mensuales del Instituto Nacional de Medicina Legaly Ciencias Forenses [1].

De acuerdo a la Política Nacional de Sexualidad, Derechos Sexuales y Derechos Reproductivos 2014-2021 (PNSDSDR) [18], la cual se fundamenta en un enfoque de derechos durante todo el ciclo vital, se reconocen acciones para la garantía de los derechos sexuales y reproductivos, como lo son promoción, prevención, diagnóstico, tratamiento, rehabilitación y paliación.

En esta política, las acciones de promoción y prevención se consideran mecanismos para promover la libre orientación y expresión de la sexualidad, disminuir la posibilidad de afectación a la vida, garantizar el acceso a los avances científicos disponibles, acceso de la información y apropiación de contenidos adecuados.

A pesar de la existencia de una política nacional, una problemática es la baja tasa de consulta desde el nivel de promoción y prevención, como es el caso de asesoría en planificación familiar, con un registro promedio de un millón de mujeres entre los dieciocho y cuarenta y cuatro años que consultan anualmente, y solo 171.000 mujeres menores de dieciocho años lo hace, preocupando aún más que la tasa de consulta de los hombres sea menor [18].

Lo anterior puede reflejar que, pese a los esfuerzos del sector salud, no se ha logrado una adherencia a los servicios de promoción y prevención por parte de los menores de dieciocho años, debido también a la baja educación previa que reciben en el ámbito de la promoción de la salud. Por tal razón, en la PNSDSDR se reconoce al sector educativo como agente intersectorial responsable del cumplimiento de los objetivos de la política, con el apoyo de docentes capacitados para brindar esta educación desde una perspectiva de derechos, sin generar juzgamientos ni discriminación y, de este modo, garantizar la trasmisión de contenidos de manera segura. 


\section{La influencia del embarazo, parto y puerperio en el piso pélvico}

Se entiende por piso pélvico al conjunto de tejidos conectivos y músculos capaz de contrarrestar la fuerza de la gravedad, así como el aumento de la presión intraabdominal, permitiendo así mantener la posición de los órganos pélvicos y su correcto funcionamiento [19].

Existen estudios que han identificado la relación entre el embarazo, el parto vaginal (debido al trauma de la musculatura y acciones obstétricas) y las disfunciones del piso pélvico [20-22], como pueden ser las infecciones e incontinencia urinaria, fecal y dispareunia, y así mismo el incremento de la prevalencia de estas alteraciones en el postparto [23,24].

Las afecciones del piso pélvico, como laceraciones esfinterianas, daño del tejido conectivo y de fibras nerviosas, pueden estar relacionados a los traumas de esta zona, incidiendo en la presentación de la incontinencia urinaria e impactando otros ámbitos como el social. Es acá donde la fisioterapia juega un papel importante, sirviendo como parte de la estrategia que tiene el fin de prevenir y rehabilitar [24,25] estas condiciones de salud.

Gracias a las estrategias de salud dirigidas a las gestantes, que permiten controles mensuales, el embarazo es un buen momento para enlazar a la mujer y el componente de prevención, mejorando la condición física y de salud de la materna y el feto [26,27]. Lo anterior puede lograrse por medio de la prescripción del ejercicio y rehabilitación del piso pélvico, enfocado no solo a la población objeto, sino también a la población en general.

Según Barakat et al [26], el tipo de actividades a desarrollar con la gestante, debe estar centrado en mejorar la resistencia aeróbica, la fuerza muscular leve, el equilibrio y la coordinación motriz, la flexibilidad, así como el trabajo del piso pélvico.

Derivado de estos procesos de intervención en donde se incluye la educación, se reduce el riesgo de futuras disfunciones, al aumentar la fuerza de la musculatura pélvica [24], mejorar la flexibilidad y preparar a la mujer para llevar a cabo un proceso de cuidado del piso pélvico durante y después del embarazo. Estas acciones se asocian al aumento de la tranquilidad de la gestante sobre su proceso de parto [28], facilitándolo y disminuyendo riesgos de enfermedad en el recién nacido, la violencia obstétrica, el uso de fórceps y la presencia de dolor en el coito postparto.

Por tal razón, la incorporación de un plan de entrenamiento de la musculatura pélvica podría desarrollarse como programa de promoción de la salud, debido a sus beneficios no solo durante los momentos de gestación, parto y puerperio, sino para toda la vida de la mujer, siendo necesario que dicho programa sea supervisado por un fisioterapeuta especializado [29,30].

El ideal de los servicios de salud es incluir el trabajo multidisciplinario, donde se incluya la fisioterapia de piso pélvico como ruta de prevención de disfunciones uroginecológicas. De acuerdo a Bary Berhgmans, toda intervención fisioterapéutica efectiva debe comenzar con un componente de educación [8], el cual puede desarrollarse en torno a la anatomía del sistema genital y reproductivo, los métodos de planificación, las enfermedades de trasmisión sexual, el proceso gestacional, los tipos de disfunciones, así como sus causas y prevención, y enfocar, de acuerdo a la edad, las sesiones de fisioterapia en la mejoría de la condición física actual y no solo como manejo de rehabilitación tras la instauración de una condición de salud. 


\section{Las disfunciones uroginecológicas en la mujer mayor y su prevalencia}

Factores como la baja tasa de asistencia a la consulta médica [18], derivada del desconocimiento por parte de la población, la estigmatización respecto a las condiciones uroginecológicas y la normalización de las mismas (considerar naturales pérdidas urinarias en el postparto o en la mujer adulta mayor), además de no contar con un cálculo global exacto (debido al uso de diferente terminología para las mismas condiciones de salud, las características de la población, los instrumentos de evaluación, la disponibilidad médica, el desarrollo socio-cultural, entre otros factores) [31] pueden generar un sesgo en el cálculo epidemiológico de estas patologías [32].

Pese a lo anterior, se cuenta con datos frente a la incidencia de la incontinencia urinaria en el adulto mayor, relacionada con el envejecimiento, así como el incremento de las condiciones de salud y su impacto en la calidad de vida de esta población [33]. Se estima que la incontinencia urinaria puede afectar desde el 58\% hasta el $84 \%$ de la población mayor, y su prevalencia en mujeres de edad avanzada es aproximadamente del 34\% [31].

La falta de consolidación de la información del cálculo epidemiológico puede limitar la formulación de estrategias para la detección temprana de las condiciones de salud que generan sintomatología del tracto urinario bajo, dificultando el diagnóstico oportuno. Por otro lado, la presencia de síntomas leves, en edades tempranas, no diagnosticadas a tiempo generan un subresgistro, lo que ocasiona que estas mujeres opten por cambiar sus actividades y roles sociales, adaptándose a su nueva condición de salud, sin realizar una consulta [34], aumentando así la brecha entre mujeres con y sin diagnóstico médico y generando un aumento de los síntomas.

La normalización de condiciones de salud pélvica en mujeres adultas mayores solo evidencia la falta de un seguimiento de la educación y salud sexual y de la mujer durante su ciclo vital.

Cabe resaltar que, gracias a la fisioterapia de piso pélvico, se pueden mejorar las cualidades y condiciones de la musculatura y, a través de diferentes técnicas y prescripción del ejercicio, promover hábitos, con el fin de mejorar la función de las estructuras que hacen parte del piso pélvico [35]. En este sentido, de acuerdo a la evolución de la condición de salud, la rehabilitación de piso pélvico en cualquier disfunción se caracteriza por ser la primera opción en tratamiento conservador, involucrando de manera activa al paciente. Es por esto que el manejo fisioterapéutico en disfunciones del piso pélvico, en sus diferentes modalidades de rehabilitación, ha demostrado buenas respuestas de tratamiento, generando una mejora de la condición o desaparición total de los síntomas [11,36,37].

\section{Conclusiones}

Las estadísticas presentadas en todo el documento muestran la necesidad de incluir una estrategia bien establecida de educación sexual integral, adoptada a los aspectos socioculturales del país, mantenida en el tiempo y con calidad de contenidos. También debe tenerse en cuenta la inclusión de los NNA de zonas rurales, quienes representan un mayor riesgo de deserción escolar, como lo advierte la OMS [5]. Además, dicha estrategia debe reglamentarse y adecuarse para los NNA, considerando que la etapa comprendida entre los cinco y diecinueve años es el momento de mayor aprendizaje del ser humano, en el que no solo se limita a la formación académica, sino que también se forjan sus vínculos socioafectivos, de comportamiento, personalidad y autoestima para su edad adulta $[38,39]$. 
Colombia es un país con legislación necesaria y riqueza en talento humano, lo cual podría permitir la construcción y ejecución de un programa de educación sexual dirigido a niños, niñas y adolescentes, siguiendo los lineamientos y las recomendaciones de la Organización Panamericana de Salud (OPS), la Organización Mundial de la Salud (OMS) [5] y la Asociación Mundial de Sexología (WAS) [6].

Pese a la normatividad existente, se evidencia una debilidad por parte del estado colombiano frente a la educación sexual y la educación en salud, de forma estructurada y transversal con los sectores de educación y salud, entre otros.

Siguiendo las recomendaciones de las diferentes entidades internacionales $[4-6,40]$, y realizando el ajuste necesario para llevar a cabo estas intervenciones en Colombia y sus diferencias culturales, por región y rangos de edad, esta estrategia puede incluir la participación de profesionales de la salud cualificados, que trabajen en conjunto con las familias y la comunidad, sin olvidar que son participes importantes en el desarrollo de los menores, y que toda la responsabilidad no recaiga sobre las instituciones educativas [41].

Un programa de educación sexual, impartido desde el currículo, podría disminuir los factores de riesgo de abuso sexual, promover la adquisición de conocimiento adecuado y el uso de fuentes fidedignas. Además, permitiría el acompañamiento médico adecuado para orientar el inicio de la vida sexual en un proceso seguro y consciente, así como la expresión de la sexualidad con libertad, al ser concebida como un derecho.

De igual manera, se podría promover el autoconocimiento como un factor de autoestima y de atención a la salud [6]. Por último, la concepción de que la atención médica en relación a la salud sexual y reproductiva también incluye temas relacionados al piso pélvico, tanto en mujeres como en hombres.

El desarrollo de este tipo de estrategia educativa apunta además al aumento de las consultas de promoción de la salud y prevención de la enfermedad, brindando un seguimiento a la salud sexual y reproductiva, así como la detección temprana de disfunciones uroginecológicas, el inicio oportuno de tratamientos y la reducción de factores de riesgo que puedan afectar la calidad de vida de las mujeres y de los hombres en los diferentes ciclos de vida.

Es por lo anterior que la fisioterapia de piso pélvico debe involucrar la educación, como una estrategia para que las mujeres aprendan a identificar factores de riesgo y signos clínicos que se traduzcan en la presencia de una posible disfunción, y así poder disminuir los subregistros, mejorar las consultas y lograr un diagnóstico y tratamiento oportuno.

Por último, considerando la importancia de este contenido y los próximos cambios en las políticas públicas, se espera que haya otras investigaciones que apunten a realizar seguimientos de las estrategias del país en relación a las políticas en salud sexual y reproductiva de las mujeres, adolescentes y niñas. 


\section{Referencias}

1. Instituto Nacional de Medicina Legal y Ciencias Forenses. Boletines Estadísticos Mensuales [Internet]. Cifras Estadísticas. 2020 [cited 2021 Mar 3]. Disponible en: https:/ /www. medicinalegal.gov.co/cifras-estadisticas/boletines-estadisticos-mensuales

2. DANE. Encuesta de Comportamientos y Actitudes sobre Sexualidad en Niñas, Niños y Adolescentes Escolarizados. 2014 [Internet]. Bogotá: DANE; 2016. Disponible en: https://www.dane.gov.co/files/investigaciones/boletines/ecas/bol_ecas_2014.pdf

3. DANE. Estadísticas Vitales (EEVV) Nacimientos en Colombia [Internet]. Bogotá: DANE; 2021. Disponible en: https://www.dane.gov.co/index.php/estadisticas-por-tema/demografia-y-poblacion/nacimientos-y-defunciones

4. UNESCO. Orientaciones tecnicas internacionales sobre educación en sexualidad. Un enfoque basado en evidencia [Internet]. Segunda edición. París: UNESCO; 2018. Disponible en: https://unesdoc.unesco.org/ark:/48223/pf0000265335

5. Organizacion Mundial de la Salud. Recomendaciones de la OMS sobre salud y derechos sexuales y reproductivos de los asolescentes [Internet]. Geneva: OMS; 2019. Disponible en: https://apps.who.int/iris/bitstream/handle/10665/312341/97892435146 04-spa.pdf?ua $=1$

6. Organización Mundial de la Salud (OMS), Organización Panamericana de Salud (OPS), Asociación Mundial de Sexología (WAS). Promoción salud sexual de la Recomendaciones para la acción [Internet]. Antigua Guatemala: OMS; 2000. Disponible en: https:// www.paho.org/hq/dmdocuments/2009/promocion_salud_sexual.pdf

7. Thiel R, Thiel M, Dambros M, Riccetto C, López V, Rincón ME, et al. Evaluación de la función sexual femenina antes y después de un procedimiento para la corrección de la incontinencia urinaria de esfuerzo. Actas Urológicas Españolas [Internet]. 2006;30(3):315-20. Disponible en: https://www.sciencedirect.com/science/article/abs/ pii/S0210480606734465

8. Berghmans B. Physiotherapy Assessment to Identify the '5Fs': A Concept of Innovative Pelvic Floor Training. Int J Phys Med Rehabil Physiother [Internet]. 2020;8(4):1-3. Disponible en:: https://www.longdom.org/open-access/physiotherapy-assessment-to-identify-the-lsquo5-fsrsquo-a-concept-of-innovative-pelvic-floor-training-54492.html

9. Bo K, Berghmans B, Morkved S, Van Kampen M. Pelvic floor and exercise science. In: Evidence-Based Physical Therapy for the Pelvic Floor: Bridging Science and Clinical Practice: Second Edition. Edinburgo: Churchill Livingstone; 2015. p. 111-30.

10. Talasz H, Himmer-Perschak G, Marth E, Fischer-Colbrie J, Hoefner E, Lechleitner M. Evaluation of pelvic floor muscle function in a random group of adult women in Austria. Int Urogynecol J. 2008;19:131-5.

11. Espitia De La Hoz FJ, Orozco Gallego H. Evaluación de la mejoría de la sexualidad en mujeres intervenidas por incontinencia urinaria. Rev Peru Ginecol y Obstet [Internet]. 2017;63(4):537-46. Disponible en: http://www.scielo.org.pe/pdf/rgo/v63n4/ a04v63n4.pdf 
12. Kandadai P, O'Dell K, Saini J. Correct performance of pelvic muscle exercises in women reporting prior knowledge. Female Pelvic Med Reconstr Surg. 2015;21(3):135-40.

13. Congreso de la República de Colombia. Ley 115 de 1994 [Internet]. Colombia; 1994 p. 4-5. Disponible en: http://www.secretariasenado.gov.co/senado/basedoc/ ley_0115_1994.html

14. Congreso de la República de Colombia. Ley 1146 de 2007 [Internet]. Colombia; 2007. Disponible en: https://www.funcionpublica.gov.co/eva/gestornormativo/norma.php?i=25669

15. Ministerio de la Protección Social de Colombia. Decreto 2968 de 2010 [Internet]. Colombia; 2010. Disponible en: https://www.icbf.gov.co/cargues/avance/docs/decreto_2968_2010.htm

16. Congreso de la República de Colombia. Ley 1620 de 2013 [Internet]. Colombia; 2013. Disponible en: http://www.secretariasenado.gov.co/senado/basedoc/ ley_1620_2013.html

17. Calle Gorrea MV, Guerrero Pérez LG, Linares Cantillo A, Mendoza Martelo GE, Ortiz Delgado GS, Palacio Palacio JI, et al. Sentencia C-085/16 [Internet]. Corte Constitucional de la República de Colombia. Colombia; 2016 [cited 2021 Mar 3]. Disponible en: https://www.corteconstitucional.gov.co/relatoria/2016/C-085-16.htm

18. Ministerio de Salud y Protección Social. Política Nacional de Sexualidad. Derechos Sexuales y Derechos Reproductivos [Internet]. Colombia; 2010. Disponible en: https:// www.minsalud.gov.co/sites/rid/Lists/BibliotecaDigital/RIDE/DE/LIBRO POLITICA SEXUAL SEPT 10.pdf

19. Easley DC, Abramowitch SD, Moalli PA. Female Pelvic Floor Biomechanics: Bridging the Gap. Curr Opin Urol [Internet]. 2018;27(3):262-7. Disponible en: https://pubmed. ncbi.nlm.nih.gov/28267057/

20. Castro RA, Santisteban Alba S, Delgado Peruyera L, Fernández Maderos I. Defectos del suelo pélvico y su impacto en la calidad de vida de la mujer. Rev Cuba Med Gen Integr [Internet]. 2017;33(2):171-9. Disponible en: http://scielo.sld.cu/scielo.php?script=sci_ arttext\&pid=S0864-21252017000200002

21. Guzmán R, Lecannelier J, Wenzel G, Descouvieres C, Sandoval C, Bernier P, et al. Factores de riesgo y prevención de la disfunción del piso pélvico. Rev Electrónica Científica y Académica Clínica Alem [Internet]. 2019;9(2):27-33. Disponible en: https://contactocientifico.alemana.cl/index.php/cc/issue/view/43

22. Araujo Da Silva BC, Amorim D, Carneiro Nunes EF, Sutter Latorre GF. Disfunção Sexual Feminina e Parto Normal: uma revisão integrativa da literatura. Rev Bras Ciências da Saúde. 2017;21(4):363-8.

23. Junqueira Vasconcellos de Oliveira SM, De Souza Caroc A, De Paula Batista Mendes E, Guimarães de Oliveira S, Penha Silva F. Disfunciones del suelo pélvico en mujeres primíparas después del parto. Enfermería Glob. 2018;17:26-39. 
24. Cohen-Quintana C, Carrasco-Portiño M, Manríquez-Vidal C, Bascur-Castillo C. Fortalecimiento de la musculatura del piso pélvico en gestantes en control en un centro de salud familiar: Un estudio experimental. Rev Chil Obstet Ginecol [Internet]. 2017;82(5):471-9. Disponible en: https://scielo.conicyt.cl/scielo.php?pid=S0717-75262017000500471\&script=sci_arttext\&tlng=en

25. Castro Adrada LM. Entrenamiento del suelo pélvico en incontinencia urinaria durante el embarazo y postparto vaginal. FisioGlia [Internet]. 2017;4(3):53-6. Disponible en: https://dialnet.unirioja.es/ ejemplar/466280

26. Barakat R, Díaz-Blanco A, Franco E, Rollán-Malmierca A, Brik M, Vargas M, et al. Guías clínicas para el ejercicio físico durante el embarazo Clinical guidelines for physical exercise during pregnancy Correspondencia. Prog Obs Ginecol [Internet]. 2019;62(5):464-71. Disponible en: https://repositorio.comillas.edu/xmlui/handle/11531/46065

27. Alejo Holgado A, Lozano Mayor L. Ejercicio físico y embarazo. Repercusión en el feto y el recién nacido. Nuberos Científica [Internet]. 2016;3(19):34-8. Disponible en: https:// dialnet.unirioja.es/servlet/articulo? codigo $=7498130$

28. Gómez Abero AM, Serrano M, Díaz D, Machicado M, Ballesta A, Hernández de Campos A. Restablecimiento del suelo pélvico en el postparto. Rev Enfermería Docente. 2020;112:83-9.

29. Palacios López A, Díaz Díaz B. Fisioterapia en incontinencia urinaria de embarazo y posparto: una revisión sistemática. Clin Invest Ginecol Obstet [Internet]. 2016;43(3):122-31. Disponible en: https://www.sciencedirect.com/science/article/abs/ pii/S0210573X15000556

30. Encabo-Solanas N, Torres-Lacomba M, Vergara-Pérez F, Sánchez-Sánchez B, Navarro-Brazález B. Percepción de las puérperas y de los profesionales sanitarios sobre el embarazo y el parto como factores de riesgo de las disfunciones del suelo pélvico. Estudio cualitativo. Fisioterapia. 2016;38(3):142-51.

31. Mostafaei H, Sadeghi-Bazargani H, Hajebrahimi S, Salehi-Pourmehr H, Ghojazadeh M, Onur R, et al. Prevalence of female urinary incontinence in the developing world: A systematic review and meta-analysis - A Report from the Developing World Committee of the International Continence Society and Iranian Research Center for Evidence Based Medicine. Neurourol Urodyn. 2020;1(24):489-512.

32. Abrams P, Andersson KE, Apostolidis A, Birder L, Bliss D, Brubaker L, et al. 6th International Consultation on Incontinence. Recommendations of the International Scientific Committee: Evaluation and treatment of urinary incontinence, pelvic organ prolapse and faecal incontinence. Neurourology and Urodynamics. 2018;37(7):2271-2271. doi: https://doi.org/10.1002/nau.23551

33. Chiang H, Valdevenito R, Mercado A. Incontinencia urinaria en el adulto mayor. Urinary incontinence in elderly adults. Rev Médica Clínica Las Condes [Internet]. 2018;29(2):232-41. Disponible en: https://www.sciencedirect.com/science/article/pii/ S0716864018300324\#bib0015 
34. Carreño RC, María L, Angarita-Fonseca, Pinto A, Lucía A, Delgado A, et al. Calidad de vida relacionada con salud e incontinencia urinaria en mujeres con exceso de peso de Bucaramanga, Colombia. Rev Ciencias la Salud. 2015;13(1):63-76.

35. Fundación Santa Fé de Bogotá. ¿Cuál es el rol de la fisioterapia de piso pélvico? [Internet]. Bogotá: Fundación Santa Fé de Bogotá . 2019 [cited 2021 Apr 11]. Disponible en: https://acortar.link/Cbd6j8

36. Alves PGJM, Nunes FR, Guirro ECO. Comparison between two different neuromuscular electrical stimulation protocols for the treatment of female stress urinary incontinence: a randomized controlled trial. Brazilian J Phys Ther. 2011;15(5).

37. Martínez Torres J del C. Caracterización de mujeres en edad mediana con incontinencia urinaria y respuesta al tratamiento rehabilitador. Rev Cuba Obstet y Ginecol [Internet]. 2014;40(1):102-18. Disponible en: http://scielo.sld.cu/scielo.php?script=sci_arttext\&pi$\mathrm{d}=\mathrm{S} 0138-600 \mathrm{X} 2014000100012$

38. Tabernero C, Serrano A, Mérida R. Estudio comparativo de la autoestima en escolares de diferente nivel socioeconómico. Psicol Educ. 2017;23(1):9-17.

39. Díaz Falcón D, Fuentes Suárez I, Senra Pérez N de la C. Adolescencia y autoestima: su desarrollo desde las instituciones educativas. Conrado. 2018;14(64):98-103.

40. International Planned Parenthood Federation. Framework for Comprehensive Sexuality Education 2010 [Internet]. Framework for Comprehensive Sexuality Education 2010. 2010. Disponible en: https://www.ippf.org/sites/default/files/ippf_framework_for_ comprehensive_sexuality_education.pdf

41. The United Nations Population Fund. Comprehensive Sexuality Education: Advancing Human Rights, Gender Equality and Improved Sexual and Reproductive Health [Internet]. Bogotá, Colombia: UNFPA; 2010. Disponible en: https://www.unfpa.org/ es/node/9591 